\title{
Chants et jeux chantés pour enfants en qene drehu
}

L'expression d'un répertoire en renouvellement permanent

\section{Stéphanie Geneix-Rabault}

\section{(2) OpenEdition}

Journals

Édition électronique

URL : http://journals.openedition.org/jso/629

DOI : $10.4000 /$ jso. 629

ISSN : $1760-7256$

Éditeur

Société des océanistes

Édition imprimée

Date de publication : 1 décembre 2006

Pagination : 187-206

ISSN : 0300-953x

Référence électronique

Stéphanie Geneix-Rabault, "Chants et jeux chantés pour enfants en qene drehu », Journal de la Société des Océanistes [En ligne], 122-123 | Année 2006, mis en ligne le 01 décembre 2009, consulté le 19 avril 2019. URL : http://journals.openedition.org/jso/629 ; DOI : 10.4000/jso.629 


\section{Chants et jeux chantés pour enfants en qene drehu ${ }^{1}$. L'expression d'un répertoire en renouvellement permanent}

par

Stéphanie GENEIX-RABAULT*

\begin{abstract}
RÉSUMÉ
Cet article, à partir d'entretiens de type ethnographique, d'observation directe et participante et d'analyse historiographique des données archivistiques disponibles, examine les questions de renouvellement et de permanence dans le répertoire que les Lifou adressent aux enfants. J'analyse les principes d'unité, de diversité, de collectif, de particularismes, de tradition et de modernité, d'une société, productrice de cette musique, en pleine mutation. La pratique de ce répertoire s'inscrit dans la tradition, mais tend néanmoins à assimiler et à relater des faits très contemporains, l'insérant alors dans la modernité. C'est pourquoi il reflète une surimpression d'apports hérités du passé et de l'actualité de chacune des interprètes.
\end{abstract}

MoTS-CLÉS : chants pour enfants, ethnomusicologie, identité, changement et continuité, tradition et modernité, Lifou.

La chanson de tradition orale a bien souvent été considérée à tort comme l'expression d'un répertoire figé, comme la répétition immuable, à l'identique, de l'héritage culturel issu des générations précédentes. En fait, elle fonctionne plutôt comme un système très ouvert, évolutif, capable

\begin{abstract}
This article, based on ethnographics interviews, direct and active observations, and historiographic analysis of the available archived datum, examines the renewal and continuity questions in the nursery rhymes adressed by Lifou people to their children. I analyse the principles of unity, diversity, community, particularism, tradition and modernity, of a society producing this music in a deep mutation. The practice of these nursery rhymes is part of tradition, but nevertheless tends to integrate and relate some very contemporary facts, inserting them in modernity. That's the reason why it reflects a double exposure of elements inheriting from the past and of the actuality of each woman's current events.
\end{abstract}

KEYWORDS: nursery rhymes, ethnomusicology, identity, change and continuity, tradition and modernity, Lifou. 


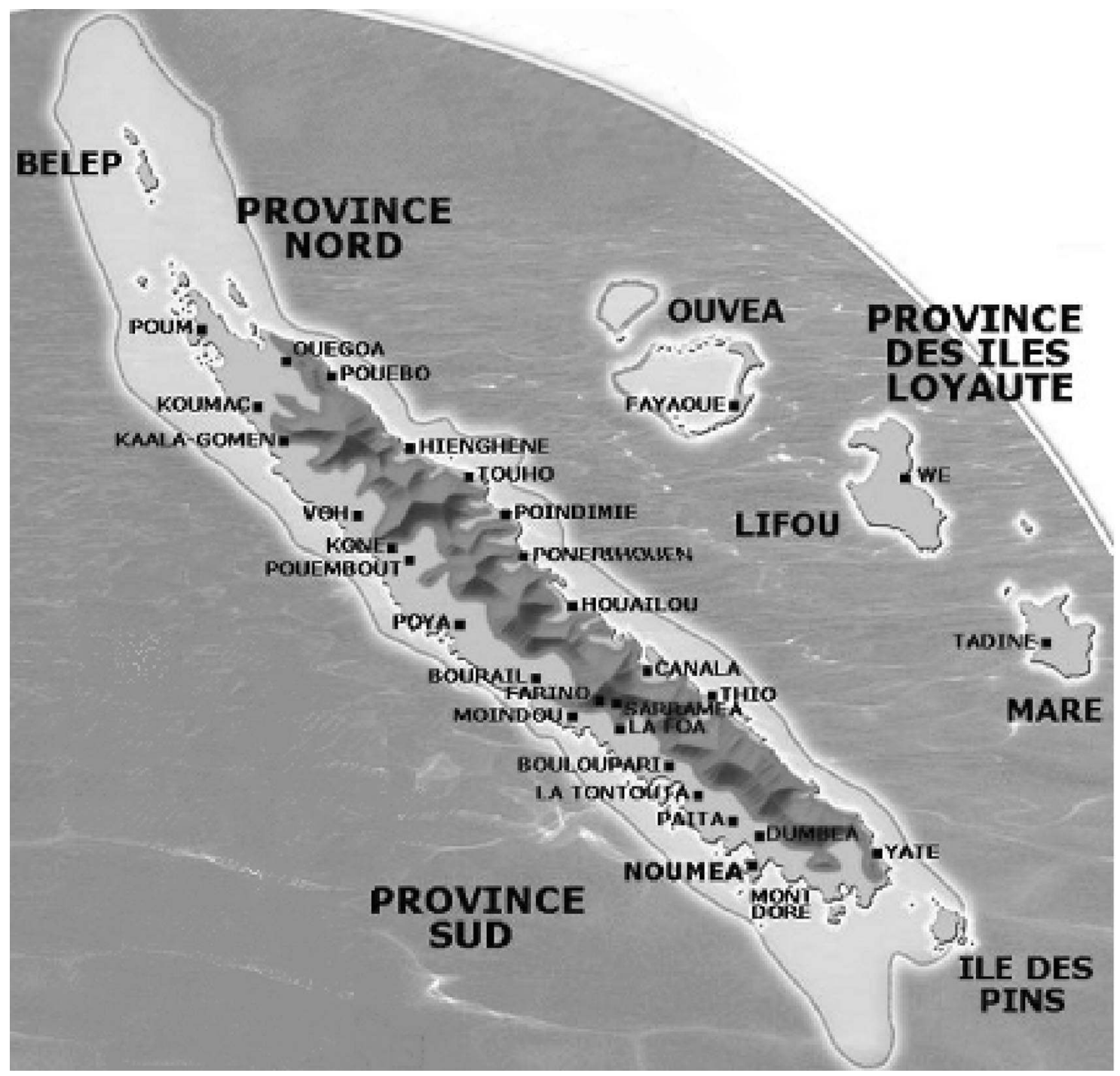

CARTe 1. - La Nouvelle-Calédonie (C Service topographique, PIL, 1996)

\section{Présentation}

J'ai effectué quatre missions ${ }^{2}$ en NouvelleCalédonie depuis cinq ans, représentant au total dix-huit mois d'enquête de type ethnographique sur ce terrain. La diversité linguistique ${ }^{3}$ m'a conduite à réduire mon champ d'investigation à l'île de Lifou ${ }^{4}$, mais j'ai néanmoins élargi mon sujet aux chants et jeux chantés pour enfants en drehu. Ma recherche de doctorat en ethnomusicologie s'intéresse aux Nyima me elo thatraqai haa nekönatr ngöne la qene drehu : chants et jeux chantés pour enfants en lifou. Les différentes investigations menées sur ce terrain depuis cinq ans m'ont permis de collecter de multiples informations sur ce répertoire et les pratiques de maternage, dont cinq cents chants et jeux chantés pour enfants. Loin de pouvoir présenter une

2. Une première investigation de six mois, d'octobre à mars 2001, a été menée dans le cadre de la préparation de ma maîtrise sur les Berceuses des îles Loyauté. Une seconde enquête, de février à avril 2002, a été effectuée dans le cadre de mon DEA sur Les chants enfantins de Lifou. Mes pérégrinations se sont poursuivies dans le cadre de la préparation de mon doctorat d'ethnomusicologie-musicologie. J'ai alors effectué une mission plus longue de huit mois, d'octobre à mai 2004 en tant que jeune chercheur associée et financée par l'ADCK et la Province des Îles Loyauté ; puis une dernière recherche de vérification des hypothèses de fin décembre 2004 à mars 2005, financée par Paris IV-LACITO-CNRS.

3. Les îles Loyauté comptent quatre langues vernaculaires différentes : le iaai et le fagauvea à Ouvéa, le drehu à Lifou, et le nengone à Maré. Les habitants de Tiga parlent indifféremment le drehu et le nengone.

4. Une des quatre îles Loyauté de la Nouvelle-Calédonie : avec Maré au sud, Tiga, Lifou au centre, et Ouvéa au nord. Ces îles coralliennes se situent à environ cent dix kilomètres de distance de la côte Est de la Grande Terre, sur une ligne nord-est sud-ouest. 
étude exhaustive sur ce sujet, la somme de données recueillies me permet d'en présenter ici quelques caractéristiques.

Dans une conception anthropologique musicale développée par Alan Parkhurst Merriam, John Blacking et André-Marie Despringre, j'envisage chaque expression musicale en la replaçant systématiquement dans son contexte historique, social, culturel et géographicoethnique. Aussi, le groupe producteur de cette musique et son interprétation sont-ils indissociables dans l'analyse de ce répertoire. Exclusivement véhiculé par les femmes de Lifou, il reflète une partie de leurs préoccupations actuelles et structure progressivement l'enfant en lui transmettant les éléments culturels dont il aura besoin par la suite une fois adulte. Les femmes d'aujourd'hui se partagent entre deux cultures : mélanésienne et européenne, tantôt plus proches de l'une que de l'autre. Rarement en rupture avec la culture d'origine, elles se situent plutôt en superposant les deux comme le note Marie Lepoutre au sujet du «pluralisme médical à Lifou » :

« On saisit [...] la nécessaire conformité, l'inévitable adhésion, mais aussi le besoin de négocier avec deux univers de discours, de pratiques, chacun ayant une légitimité et un pouvoir contraignant et suggestif très puissants sur l'individu et le groupe. [...] Certains entretiens confirment largement cette vision très clairvoyante des allers-retours entre différentes cultures, des relations de miroirs. Le contact et, par là même, la confrontation, amènent de nouvelles perceptions de soi-même et créent de nouveaux espaces.[...] En définitive, accoucher au dispensaire ne signifie pas toujours accoucher à la française, consulter une sagefemme n'exclut pas la visite d'une spécialiste kanak en matière d'obstétrique. La forte incidence de la médecine occidentale n'indique pas que celle-ci se substitue à la médecine locale. » (Lepoutre, 2000 : 302-306)

Leur rôle social au sein de la société a évolué, tout comme leurs préoccupations. C'est pourquoi elles véhiculent, au travers de ce répertoire, une identité culturelle, des racines, une histoire collective et/ou individuelle, ancienne et/ou contemporaine. Ce répertoire se caractérise par une certaine part de diversité et d'unité, entre variations infinies et fidélité. Il s'inscrit comme la formulation d'une certaine forme de «tradition» et de «modernité ». Il se compose d'expressions musico culturelles en renouvellement permanent.

\section{Entre histoire collective-individuelle, ancienne- contemporaine}

Les chants et jeux chantés que les femmes adressent aux enfants se présentent comme le lieu d'expression privilégié d'une mémoire historique orale aussi bien collective qu'individuelle, ancienne et récente. Ces repères et indices chronologiques sont précieux pour l'analyse de l'évolution de cette société.

Par histoire collective, il faut comprendre tous les événements communs à l'ensemble des Lifou, une lecture globale et entièrement partagée par tous les habitants de cette île, qu'elle soit très ancienne ou contemporaine. La berceuse Ulili sine dreli ( $\ll$ Le petit Dreli gronde ») relate en qene miny ${ }^{5}$ "le chemin des diables d'avant » (gojeinyip en qene drehu), c'està-dire le chemin d'origine et les différentes pérégrinations des premiers habitants de Lifou dans les trois districts.

\section{Ulili sine dreli keikei hnaopë theng}

«Le petit Dreli gronde chancelle la caverne est à moi. $»^{6}$

\section{Ou cette interprétation plus longue :}

Manyai waminya unemi pulu goeën hmahma ju kö Zeula loe Alaxutren a tha wedrë ni kö me angetre mangalu. Itre ka hohopatr me ina getre pen. Hnine uni e sepel lo qëmekene pi hanying pi föenge hnenge hna goeën i hoima wawange ju hë muj lapa sai xötreng ju itreqe ie hae wenge $i$ a tro. Ulili sine dreli keikei hnaopë theng. Wiëhao he itreinge pe hë itra manyia tha hna xu onone a ihu hne kuje kö e keuë e wanek a zi wanaza e ngöne hna kungözing lue iweng a tro nge tro elë jë e hna nyi sinatutr me hna eli catrei nge tro elë fë ni e hune i mesup a të wai hanying a ihnyima ju hi ho menumenu lue meken ha nekönatr kei qeipië a xepë canadro. Temoni a tro nango lapa qae dreli a pua hane kuë gojenyi atre qeji tro ie hae wenge iatro. ${ }^{7}$

La comptine Titiriofa (« Á bord du Triomphant ») véhicule à la fois une histoire locale, territoriale et plus globalement internationale. Celle-ci relate l'arrivée du bateau du général de Gaulle sur l'île et sur le territoire calédonien au moment de la Seconde Guerre mondiale. Les Lifou, et d'autres Kanak de Nouvelle-Calédonie, sollicités par les grands-chefs, à la demande du général français, ont embarqué sur ce bateau pour aller se battre contre les Japonais.

Ti Tiriofa lo he i Dögol. Goe pë hë Zapo simesime hë Tiriofa. Öni Mikado sepalapen troa iwej.

5. Ancienne langue cérémonielle utilisée pour s'adresser aux grands-chefs et à toute personne de haut rang.

6. Je remercie très sincèrement Léonard Drilë Sam pour son fidèle soutien et son accompagnement dans mes recherches, par ses lectures, ses corrections et ses commentaires avertis sur les transcriptions-traductions en drehu. Oleti atraqatr !

7. Le sens de cette « enfantine » (voir note 8) en qene miny n'a pas pu être traduit littéralement, soit parce que les interprètes en ignorent le sens, soit parce qu'ils n'ont pas voulu me le divulguer. Seul le contenu général a été explicité. 
« À bord du Triomphant, le bateau de de Gaulle. Avant que le Japon ne fasse quoi que ce soit, le Triomphant est déjà loin. Ce n'est pas la peine de le poursuivre, déclare le Mikado. »

Cette « enfantine ${ }^{8}$ » peut aussi se retrouver sous cette forme :

Ti Tiriofa la he i Dögol. Xei pë hë Zapo simesime hë Tiriofa. Öni Mikado sepalapen troa kuqan. Öni Dögole qatr ase hnyawa ha la isi.

« À bord du Triomphant, le bateau de de Gaulle. Avant que le Japon ne se retourne le Triomphant est déjà loin. Il ne servira à rien de le bombarder, dit le Mikado. La guerre est bien terminée, dit le vieux de Gaulle. »

Enfin, d'autres enfantines parlent de faits plus contemporains, comme l'arrivée du premier avion sur l'île, avec la chanson Avio $i \mathrm{TeAi}$ ("L'avion de [la compagnie] TAI [Transports aériens intercontinentaux]»), qui raconte l'apparition du premier appareil de transport aérien intercontinental de marchandises. Les avions de la compagnie TAI atterrissaient surtout à la Tontouta. Les habitants de Lifou ne les voyaient que lorsqu'ils passaient dans le ciel :

Avio i TeAI kola ujëne la fen kola thipithipi lai wene rezë.

«Les avions de [la compagnie] TAI ont changé le monde. Les grappes de raisins pendent. »

Ainsi, différentes enfantines font non seulement référence à des faits historiques concernant l'ensemble de l'île, et plus largement du territoire, mais elles abordent également le contexte historique international.

L'évocation d'événements ne se résume pas seulement à des repères chronologiques admis par tous les Lifou. D’autres chansons véhiculent une mémoire historique plus individuelle, aussi bien ancienne que contemporaine, concernant seulement certains districts, certains clans. La berceuse Aköne Caeё... ( « Les clans de la chefferie $»)^{9}$ énumère le lieu d'émergence et les pérégrinations des différents clans constituant le Lösi, jusqu'à leur position actuelle, expliquant ainsi les relations entre les différents groupes de ce district.

Aköne Caeё me Nekö i Sinepi Waheo Wahile Watreudro, Pia Wahnyamala me angetre Lösi, ekölöhini Wahemunemë. Lapa neköeng pëhë angetre Lösi, pë loi angatr, pë tixe i angatr, pë titaxapo ne la baselaia $i$ nyidrë, ekölöhini Wahemunemë. Tha hna majemine troa upi nyidrë, kowe la huliwa matre iananyi. Ekölöhini hekölö i hekölö, hekölöhini Wahemunemë.
« Aköne, Caeë, et Nekö i Sinepi, Waheo, Wahile, Watreudro, Pia, Wahnyamala, les gens de Lösi. Disons au revoir à Wahemunemë. Les gens de Lösi restent orphelins, sans joie, sans chef. La chefferie reste sans protection. Oh, Wahemunemë. On n'a pas l'habitude de le voir éloigné de nous par le travail. Oh, oh, oh, oh, Wahemunemë. »

La berceuse Cai wamine $t u^{10}$ (« Une petite est née ») célèbre l'anniversaire de la fille du grandchef de Gaïca. Autant de références propres à un seul district. D'autres enfantines dispensent des repères géographico-historiques propres à un clan.

Ca $\mathrm{i}$ wamine tu e calojë e Jope a xome la wakae. Alo alo Zawe ekölö iaue. Lozati joxu hane hi lo la nyima ne atrunyi nyipëti, alo alo Zawe ekölö iaue. Angetre Gaïca me angatresi lu'atresi hane hi lo la joxu ne tro së a thili kow i lis i rouz i kuron ka lolo.

«Une petite est née là à Jope vers le Sud. Oh Zawe, oh Zawe. Princesse Loza, voici un chant pour t'honorer, oh Zawe, oh Zawe. Gens de Gaïca et vous les atresi [protecteurs de la chefferie], voici notre princesse, celle devant qui nous devons nous humilier, une fleur de lys, une rose, une couronne magnifique. »

La berceuse Koma saja (« Les pérégrinations ») retrace les pérégrinations amoureuses de deux jeunes filles, Sesile et Aleva, d'un clan de Hnathalo dans le Wetr. D'après les paroles, la chanteuse s'adresse à ces deux jeunes filles pour leur raconter ses pérégrinations amoureuses :

Koma saja lapa ju pe lue jajinyi Sesile me Aleva. Matre qaja jë ni lo itre none hanying. E cailo nöjeng. Ngo ametre kö $i$ Siling eje hi e qëmekeng sine celo i hning a $i$ wenethëhming Silinge ka menu kame (X2). Koloe Sineze kolepi e Gaica. Hnimikone e Thithë. Kelati atre We. Pujene atre Cila. Sosi e Hnathalo. Hmuine e Ejengen. Xenieti e Qasa. Oel e Jokin. Ixe e Nonime. Anane atre Ewë. Jone atre Ladran. Cumë ka idreuth. Saulo atre Kone. Jesi atre Jepo. Xulu qa Hnamenë. Nakoa qane ju pe lo itre xai hanying.

«Les pérégrinations de deux jeunes filles Sesile et Aleva. Pour raconter leurs amours. Partout ailleurs dans le pays. Sine de Gaïca. Hnikone de Thithë. Kela de We. Puje de Cila. Sosi de Hnathalo. Hmuine de Ejengen. Xenie de Qasa. Oel de Jokin. Ixe de Nonime. Anane de Ewë. Jon de Ladran. Cumë qui est fiévreux. Saulo de Kone. Jesi de Jepo. Bien avant, Hnamenë. C'est toi Nakoa qui a leur amour. "

Dans le cas d'évocations de faits historiques propres à un clan, les chants et jeux chantés, que les adultes adressent aux enfants, prennent des configurations micro-localisées.

8. Le terme « enfantine » désigne, en ethnomusicologie, un ensemble de catégories de pièces adressées aux enfants, tandis que chason se rapporte à une catégorie précise de pièce.

9. Chant créé à l'occasion du départ du fils du grand chef de ce district pour l'armée.

10. Ca $i$ wamine tu signifie littéralement « un brin de brède morelle » (NDLR. - Solanum nigrum). 


\section{Entre diversité et unité}

Dès lors, ce répertoire regroupe une multitude de pièces variées, contenant des chants et jeux chantés admis et pratiqués indistinctement par tous les Lifou et d'autres plus individuels, plus localisés. Il faut alors prendre en considération aussi bien les pratiques collectives, dont l'utilisation est largement attestée sur l'ensemble de l'île, que celles plus individuelles qui, matérialisées de manières différentes, peuvent en fait correspondre à une règle totalement partagée par tous. D'autant plus que, dans la culture Lifou, comme le note l'ethnomusicologue suisse Raymond Ammann, chaque district possède son propre répertoire de danse, ainsi qu'un fonds commun à l'ensemble de l'île :

"À Lifou, aujourd'hui, chaque chefferie a un répertoire particulier de danses traditionnelles "sacrées". Parmi les danses les plus "sacrées", on compte le fehoa, le bua et le drui. Le fehoa peut être présenté par des groupes de toutes les chefferies. Le drui est bien gardé par les gens de Gaïca, tandis que le bua appartient à la chefferie Bula de Lösi. La situation dans le district du Wetr est particulière. Les danses n'ont pas été transmises, mais l'association culturelle de Wetr a tout récemment commencé à créer et à présenter des danses, que le public apprécie beaucoup. » (Ammann, 1997 : 222-223)
Ainsi, la pratique d'un répertoire pour enfants, à la fois unanime et différente en fonction de chaque district, n'est pas une attitude isolée, puisqu'elle se retrouve dans la musique de danse, autour de laquelle gravite la majeure partie de la vie musicale.

D'autres paramètres peuvent justifier cette caractéristique d'interprétation propre à un district. À travers ces chants et jeux chantés, les femmes signifient à l'enfant son appartenance à une lignée humaine, à une culture et le sensibilisent à l'histoire de ses origines. Cet héritage chanté est le principal lien communautaire, enraciné, indispensable à la vie. On se rend alors compte que ce qui varie le plus dans les chansons pour enfants, ce sont les changements de références toponymiques et anthroponymiques, puisque chaque grand-mère transmet à l'enfant sa propre histoire, ses propres repères identitaires. C'est le cas dans la formulette pour appeler l'âme des morts Thithi qau lolo (« Prier») :

Thithi qau lolo xulu jë $i . .$.

«Prier, prier pour appeler l'âme de [nom d'un ancêtre]»

ainsi que pour la formulette de jeu mimée Tra nu fe inagoj («Planter le cocotier à Inagoj») ${ }^{11}$ :
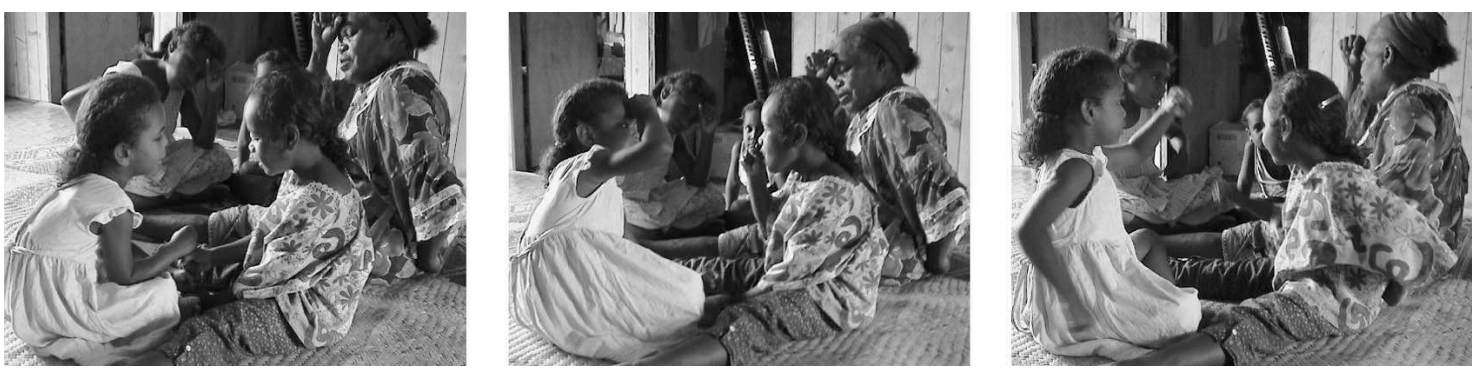

Pнотоs 1. - Famille Alikie (Hmeleck) (Clichés de S. Geneix-Rabault, 30/03/2004)
Nu i drei caha? Nung. Troa trane eka? Trao trane e .... Iji jë nge pë jë.

Tra nu fe Inagoj. Isa iji wene nun. Iji jë nge pë jë.
« À qui appartient ce cocotier? À moi. Où le planteras-tu ? Je le planterai à [nom de lieu]. Bois-le et vide-le.

Planter le cocotier à Inagoj. Chacun boit son coco. Bois-le et vide-le. [Il n'y en a plus.] » 

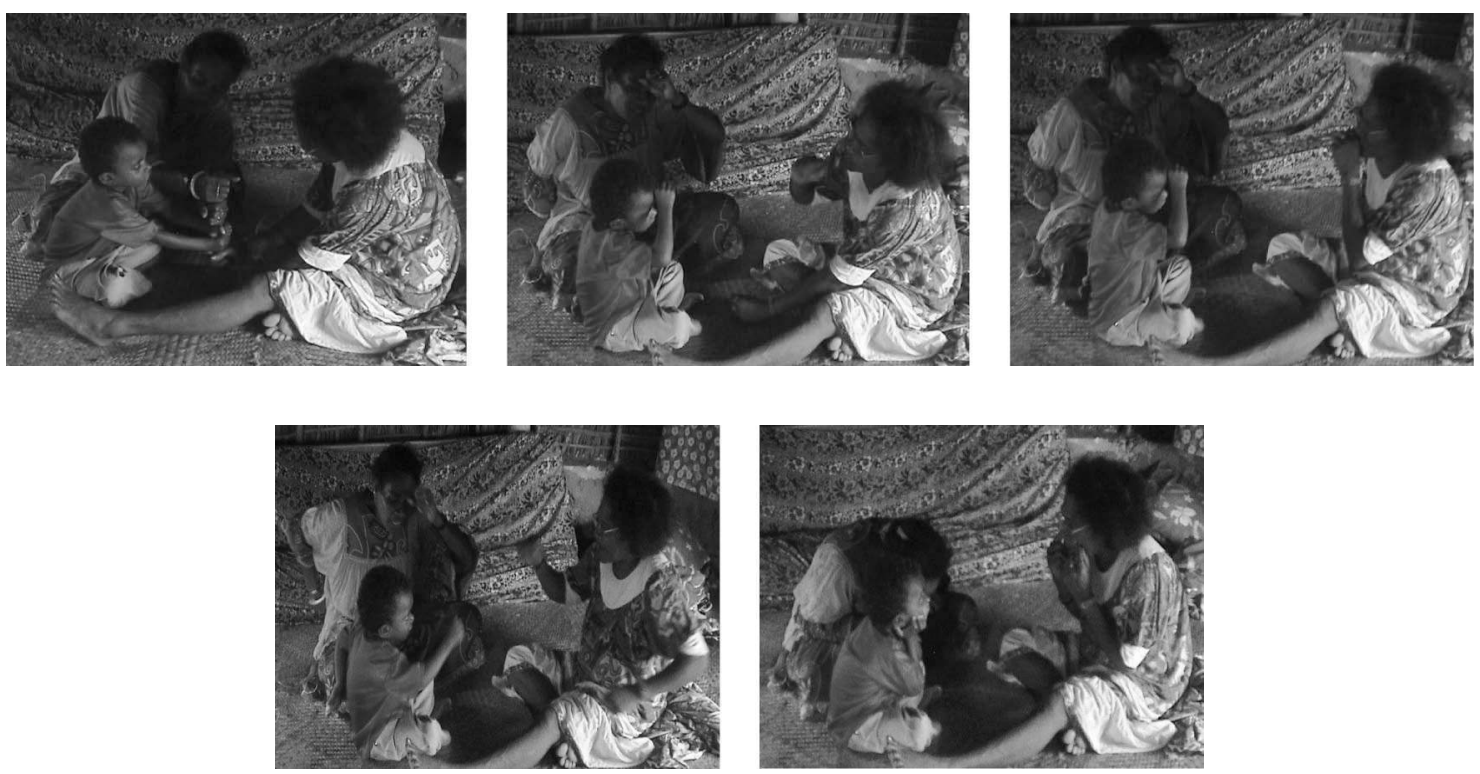

Рнотоs 2. - Famille Haluatr (Drueulu)

(Clichés de S. Geneix-Rabault, 25/02/2005)

Nu i drei caha? Nu i eni. Troa trane eka?... Xome jë nge iji jë.

$\mathrm{Nu}$ fe nu fe inagoj. Hulia alameke i qatreföe.
« À qui appartient ce cocotier? À moi. Où le planteras-tu? [Nom de lieu].

Prends-le et bois-le. Cocotier Inagoj. Les yeux exorbités de la sorcière! »
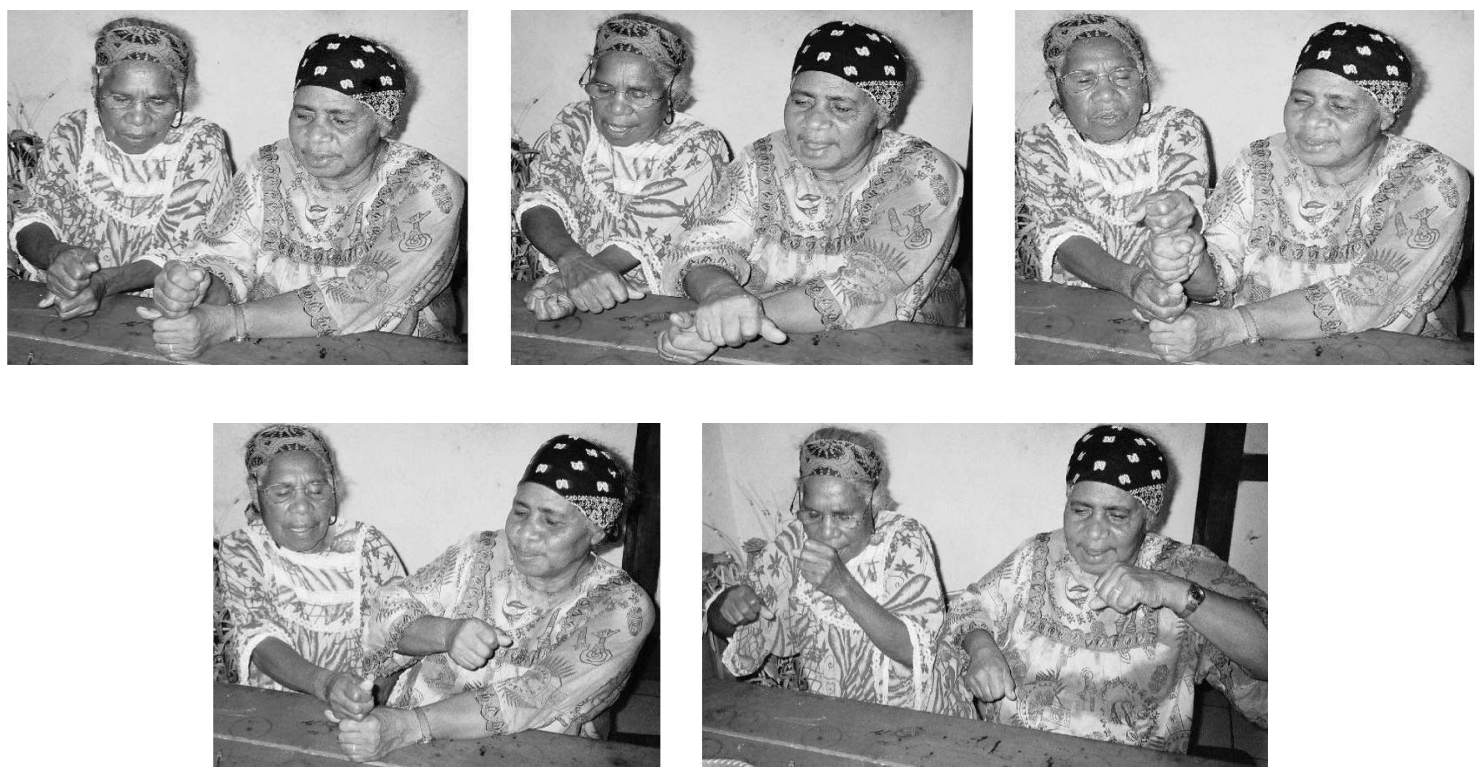

Photos 3. - Grand-mères (Tingeting)

(Clichés de S. Geneix-Rabault, 23/03/2004)

Wasuma ju ipië, koko jë draië.

Nu i drei caha? Nu i eni. Troa trane eka? ... Xome jë nge iji jë.

Ca ono pë hë kohmiju a i angetre Nganawa
« Le taro en bas. L'igname en haut.

À qui appartient ce cocotier? À moi. Où le planteras-tu? [Nom de lieu]. Prends-le et bois-le.

Il ne reste plus qu'un coco là-bas, il sera pour les gens de Nganawa. » 


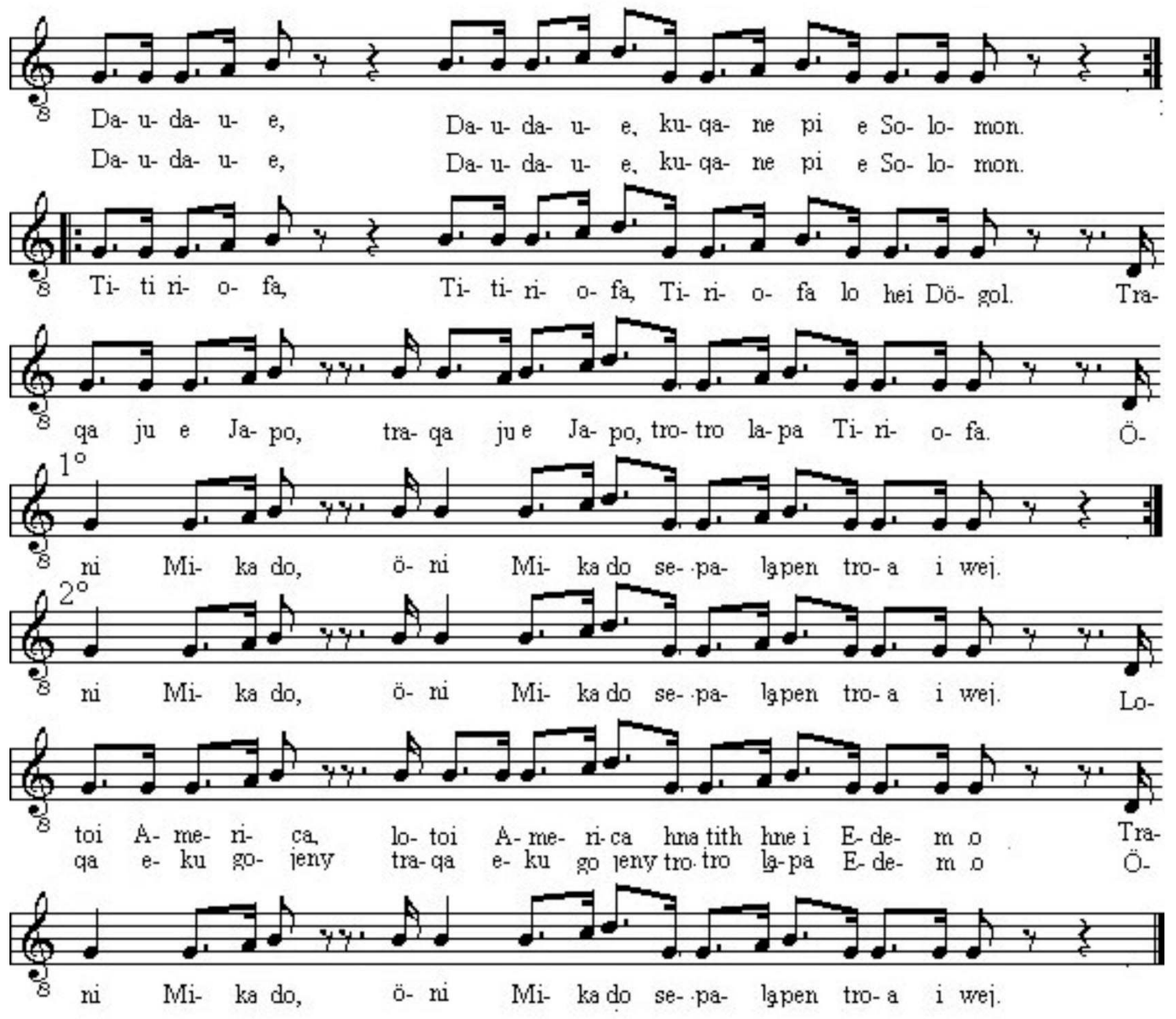

Partition $1:$ « Daudaue », Chant pour enfants, Otrenë qatr Kakue (Tingeting)

(C) Stéphanie Geneix-Rabault)

Ces variations de références textuelles personnelles font évoluer et influencent ce répertoire. Ce constat justifie en partie les diverses interprétations existantes. Mais elles engendrent par la même occasion d'autres incidences aux niveaux rythmiques, mélodiques, formels, imposées par les différents changements littéraires, comme le reflète la partition ${ }^{12}$ de l'enfantine Daudaue ci-dessous. Le texte prime sur la musique et détermine les configurations des différents paramètres constitutifs de ce répertoire :

« Le chant contient un thème musical indéfiniment répété pour faire ressortir tous les mots du texte. À chaque répétition, il faut effectuer des modifications de rythme pour permettre de bien prononcer le texte. » (Ammann, 1997 : 244).
Mais la spécificité n'exclut pas l'unicité. Inversement, il existe des pièces communes à l'ensemble de l'île, dont l'attestation est largement répandue sur tout l'archipel. Ainsi, les pièces sans contenu historique propre à un clan, sans sens précis ou cohérent, favorisant l'éveil de la motricité, peuvent circuler librement sur les trois districts, comme c'est le cas pour les formulettes de jeu mimées Eka sine hages (« Où est le mouchoir? ») ou Emo kofi (« Moudre le café »).

Eka sine hages? Kohmiju thei Madame. Madame $i$ drei? Madame i Tivolie. Eca kölö Mama ehong.

«Où est le mouchoir? Là-bas chez la dame. La dame à qui ? La dame à Tivollier. Oh Madame! »

Emo kofi. Nenge pi hë. Iji jë hë. Nenge pi hë. Eka la hnei trepe kofi? Hane hi la.

12. La transcription musicale, de type descriptive, est régie par des principes et ne prétend qu'à refléter la réalité auditive de la pièce. Aussi, face à la grand variabilité des interprétations, il est inutile que tous les détails soient rapportés. Seuls les paramètres essentiels sont transcrits. C'est pourquoi chaque pièce est transposée en sol3 pour en faciliter la comparaison et éviter la multiplication du nombre d'altérations. La mesure solfégique, établissant naturellement la place des temps forts et faibles, ne figure pas sur les transcriptions. Le principe de répétition détermine la segmentation des unités qui sont superposées. 
«Moulons le café. Servons-le. Buvons-le. Servonsle. Où posera-t-on le café ? Là. »

De plus, il est tout à fait possible d'établir que chaque grand-mère puise dans un fonds commun de références thématiques, culturelles et musicales, de schèmes inhérents aux chants et jeux chantés propres aux Lifou. Il existe donc réellement une part d'unité, dans la symbolique thématique d'inspiration, dans l'expression stylisée et codifiée caractéristique, la verbalisation de préoccupations dans lesquelles se reconnaît l'ensemble de la communauté de Lifou, malgré la diversité d'expressions possibles, constat souligné par Isabelle Bril en linguistique :

« La variation est un phénomène essentiel des langues kanak. En rendre compte évite de trop idéaliser une entité linguistique ; cela aide, au contraire, à montrer son extension et sa complexité, tout en permettant aux divers groupes qui parlent une langue de s'y reconnaître, car ces variations sont souvent le fait de réflexes identitaires [...] Variation ne signifie toutefois pas atomisation linguistique, il est possible de décrire une langue et ses variations, tout en montrant son unité. Linguistiquement, on considère qu'on a affaire à une même langue quand les structures phonologiques, syntaxiques et sémantiques ne présentent que des variations ne gênant pas l'intercompréhension. » (Bril, $2000: 279$ )

Aussi, malgré des interprétations divergentes et similaires, les Lifou identifient et reconnaissent l'ensemble des expressions musico-culturelles comme représentatives de leur identité.

\section{Entre variation et fidélité}

Outre les changements liés aux références historiques, aux nominations toponymiques et anthroponymiques, il existe ainsi dans la chanson traditionnelle une multitude d'interprétations infinies directement liée à la mémoire des informatrices. C'est pourquoi il subsiste dans ce répertoire de tradition orale des combinaisons sans cesse renouvelées, variant presque à chaque fois : entre deux femmes du même clan, de la même tribu, du même district, ou bien d'origines différentes, mais aussi entre deux interprétations successives d'une même informatrice.

Isabelle Bril, au cours d'enquêtes linguistiques menées sur le nêlêmwa ${ }^{13}$, effectue le constat suivant :

«Lorsqu'on travaille avec diverses personnes, dans les divers endroits où est parlée cette langue, on ne peut que constater l'évidence d'une très grande variation linguistique. Il faut alors analyser les phénomènes complexes qui sous-tendent ces variantes, qui sont soit aléatoires, soit systématiques. [...] Il s'agit de variantes locales ou individuelles dans une même zone linguistique [...] Parfois il s'agit de variantes qui ne sont le fait d'aucune incorrection ou erreur [...]. [Les] variantes "systématiques" [...] forment un système et relèvent plutôt de variantes dialectales. »(Bril, 2000 : 276277)

Partant du principe que la variation est l'essence même des langues vernaculaires comme de toute chanson de tradition orale, il est alors intéressant d'examiner la nature des multiples modifications contenues dans ce répertoire pour enfants. Elles se situent à différents niveaux. Certaines de ces caractéristiques sont mises en avant par Raymond Ammann au sujet des chants et jeux chantés pour enfants :

«Au début, les mots du texte sont fixes, mais en continuant la danse, de nouveaux mots peuvent être ajoutés spontanément. » (Ammann, 1997 : 216)

Il peut alors s'agir de changements et/ou de suppressions de mots et/ou de bribes de phrases et/ou de phrases entières. Dans d'autres cas, certaines enfantines commencent par le début d'une chanson et se terminent par une autre, ou bien introduisent aléatoirement des éléments puisés ailleurs. Certaines informatrices peuvent aussi enchaîner successivement plusieurs chants, dans leur intégralité et/ou par bribes. Il arrive quelquefois que, pour combler un trou de mémoire ou pour prolonger l'enfantine, certaines onomatopées se glissent dans l'interprétation. Il se peut également, qu'en fonction de la mémoire de l'informatrice interrogée, elle soit raccourcie ou rallongée à volonté. Ainsi, une interprétation plus courte et une plus longue ont pu être collectées pour une même chanson.

Outre les distorsions de longueur liées aux variations textuelles et aux questions de mémoire, d'autres paramètres viennent conférer à ces expressions chantées de tradition orale de multiples combinaisons : en raison de son caractère très fonctionnel, les enfantines peuvent être rallongées ou raccourcies à volonté, jusqu'à ce que l'effet désiré soit obtenu. Aussi, une berceuse sera interprétée jusqu'à ce que l'enfant se soit calmé ou endormi. Pour les chants et jeux chantés collectifs, il n'existe pas de durée prédéfinie, puisque leur longueur dépend en définitive du nombre de participants.

Cette énumération, non exhaustive, correspond seulement aux diverses modifications relevées dans les données collectées sur ce terrain. 
Elle permet de mettre en exergue que les variations sont multiples et infinies dans la chanson de tradition orale et qu'il existe une version presque nouvelle à chaque interprétation. Dès lors, ce répertoire pour enfants prend des tournures bariolées et sans cesse renouvelées, sans version plus fidèle ou plus juste qu'une autre. Aussi, par définition, la chanson de tradition orale fonctionne comme un système très malléable, changeant et variant, ne reposant pas sur une seule et unique interprétation.

Bien que chacune d'entre elles soit tout aussi acceptable, il faut souligner qu'à plusieurs reprises les informatrices consultées ont prétendu détenir la version originelle de cette chanson. En effet, les grands-mères se considèrent comme de bonnes représentantes de la culture et sont convaincues de connaître la seule version admise d'une pièce. D'autant plus que, dans la culture kanak, les individus respectés et renommés sont ceux qui se sont nourris de la "parole des anciens ». Et c'est souvent dans le souci de s'affirmer comme d'excellentes représentantes de leur culture que les grands-mères se sont efforcées de restituer les versions les plus « justes » et les plus «vraies » possibles. Ce critère est très largement subjectif et arbitraire. Il provient souvent d'une acceptation très personnelle de la tradition. Il sous-entend en fait que chaque informatrice considère la version qu'elle connaît comme celle communément admise par toutes. Aussi, lorsqu'une grand-mère constate des divergences d'interprétation, elle s'efforce alors avec ferveur de corriger ce qu'elle considère comme des erreurs, des fautes, en précisant bien souvent : «Ce n'est pas la vraie chanson ça », « c'est pas $^{14}$ comme ça qu'il faut faire ». Elle se charge alors immédiatement de remédier à cette erreur en exécutant la «bonne» interprétation.

La réponse à la question de l'authenticité ne m'appartient pas. Il faut néanmoins souligner, en considérant les propos des informatrices, que l'interprétation de ces expressions chantées de tradition orale pour enfants s'évertue à la permanence, à la fidélité et à la stabilité. Elle n'est pas censée inventer mais reproduire le plus fidèlement possible ce qui lui a été transmis par la génération précédente. Et pourtant, malgré une multitude de configurations possibles, tous reconnaissent et identifient les éléments comme relevant de leur propre culture, comme représentatifs de leur appartenance à un certain groupe géographico-ethnique. Aussi cet attachement à la permanence se superpose à une certaine dose de variabilité plus ou moins individuelle, puisant constamment dans un même fonds culturel commun.

\section{Entre « tradition et modernité » ${ }^{15}$}

J'aborde ici l'essence même de la chanson de tradition orale, qu'il s'agisse ou non du répertoire lifou que les adultes adressent aux enfants. Par définition, elle perpétue ainsi la reformulation d'un parfait équilibre entre apport du passé et inscription dans le présent. Les expressions collectées sur cette île reflètent une nouvelle formulation de l'héritage oral chanté issu des générations précédentes, combiné à des éléments contemporains. La notion de tradition, très longtemps considérée comme la reproduction à l'identique d'éléments transmis par les ancêtres, sous-entend des critères liés au temps. Cette terminologie $\mathrm{a}$, bien souvent à tort, été associée à des notions de passé indéfini, figé, de statisme, de répétition immuable, de conservatisme. Et pourtant, il n'en est rien, comme le précise Jonathan Friedman :

« [la] tradition est effectivement constamment soumise à la transformation tant qu'elle participe du processus de changement social mais l'on repère également des continuités significatives au sein même de la transformation. » (Friedmann, $2002: 226$ )

Ces concepts de «tradition», " traditionnel », sous-entendent une référence au temps qui suggère plutôt une fréquence d'utilisation, l'attestation de l'ancienneté d'une pratique déterminée. Pour confirmer cette qualification, il faut observer à la fois les données historiographiques et celles plus actuelles disponibles sur ce sujet. Toutes permettent d'obtenir des données sur la question de l'ancienneté et de la nouveauté des catégories de ce répertoire et témoignent de la permanence de cette pratique orale chantée. Son attestation est relevée de nombreuses fois, que ce soit dans les sources écrites (bambous gravés, récits de voyageurs, de missionnaires, littérature kanak contemporaine), iconographiques, ou dans les propos mêmes des informateurs interrogés. Tous les témoignages recueillis, par observation directe et participante et par entretiens libres ou semi-directifs à ce sujet, au cours des différentes enquêtes de terrain, viennent confirmer l'existence ancienne de ces pratiques orales chantées des adultes à l'égard des enfants. Elles peuvent être relatées de différentes manières, soit en tant que témoin oculaire des pratiques des grands-mères ou des mamans, soit 
en tant qu'acteur direct. Enfin, l'expression de certaines enfantines en qene miny témoigne également de l'ancienneté de ce répertoire. Ces différentes attestations permettent de signaler la permanence de ces pratiques et les inscrivent alors effectivement dans la tradition.

A contrario, la tradition a été nouveauté dans un passé indéfini, changement, innovation. Et les chants et jeux chantés traditionnels sont aussi capables d'utiliser, d'adapter, d'assimiler, de reproduire des apports exogènes divers tout en restant eux-mêmes. En attestent les évolutions spectaculaires de la langue vernaculaire : l'introduction et/ou la suppression de mots en drehu, la multiplication d'emprunts au français et à l'anglais. Mais d'autres paramètres comme l'expression des transformations socio-historiques reflètent une réadaptation permanente d'éléments de la culture traditionnelle au contexte local évolutif. Anna Paini souligne alors au sujet du port de la robe mission à Lifou :

«L'objectif ici est de montrer le changement culturel constant à travers le regard et la pratique des femmes kanak, dans un contexte où les éléments exogènes se transforment au niveau local, mais restent toujours perçus en interaction avec les éléments culturels endogènes. » (Paini, 2003 :234).

De plus, la culture de Lifou et les expressions musico-culturelles qui y sont associées se sont adaptées à des changements importants au cours de ces cent cinquante dernières années, en assimilant un nouveau langage musical introduit par les missionnaires, puis fixé par l'introduction d'instruments tels que la guitare, l'accordéon chromatique, l'harmonica. Elles se sont adaptées à des éléments nouveaux, tout en conservant leur propre mode de fonctionnement, comme dans $\dot{A}$ la pêche aux moules:

«À la pêche aux moules, xulu jë hi Mani qatr ca watrenge ka xulu ca kuli ka pa la pun.

À la pêche aux moules, le vieux Mani arriva avec un panier percé, suivi d'un chien sans queue. »

L'intégration du système tempéré à la langue autochtone opère un véritable enrichissement de la culture. Aussi, ce qui est considéré et relaté aujourd'hui comme un répertoire authentique et traditionnel s'appuie sur des critères bien souvent trompeurs et arbitraires. Ce qui est actuellement qualifié "d'authentique», de «traditionnel», a été un jour nouveauté, création individuelle et changement.

Ainsi, la pratique et les interprétations actuelles et celles «d'avant» se construisent à partir d'allers-retours permanents entre le passé et aujourd'hui, comme le soulignait Jean-Marie Tjibaou :

« C'est donc le temps du débat entre l'option pour la modernité et la peur de perdre son identité. Ce débat sera long et il nous faudra surmonter cette contradiction. La symbiose entre le traditionnel et le moderne s'opère en effet par la force des choses. Les nouvelles formes d'expression la réalisent par l'intégration du matériel. Les sons sortent de la guitare, mais c'est pour accompagner des thèmes poétiques ou contemporains spécifiquement mélanésiens. De même les manous (pagnes), les sifflets de rythme, les peintures et poudres décoratives, l'harmonica et les tambours utilisés aujourd'hui dans nos danses, nos pilous, matérialisent-ils cette intégration de la modernité, de l'extérieur. Nous intégrons, de manière peut-être moins nette, les éléments de cultures environnantes dans notre chorégraphie. » (Bensa, $1998: 160)$

L'expression du chant elle-même s'enrichit constamment et se réinterprète, se réajuste en permanence, au gré des changements et des évolutions. Elle assimile toutes les adjonctions successives, qu'elles soient imposées ou non, et empile différents éléments. Dans cette lente maturation, aux orientations multiples, les individus ne reproduisent pas exactement les choses telles qu'elles leur ont été transmises par les générations précédentes. Le répertoire pour enfants prend bien au contraire des configurations qui sont propres à chaque génération qui les véhicule. C'est pourquoi le tissu du corps social se réinscrit en permanence dans ces chants et jeux chantés, s'adaptant aux changements et aux évolutions auxquels les interprètes sont confrontés. Le répertoire enfantin comprend aussi bien des références aux valeurs traditionnelles, culturelles, morales et sociales admises et attestées depuis longtemps au sein de la société dans laquelle il s'insère, comme dans Fitiku kalekale, que la verbalisation de préoccupations ou de phénomènes très contemporains, comme des principes directement issus du modèle d'éducation à l'occidentale - « il faut bien travailler à l'école pour avoir un bon travail» ou encore dans la chanson Neköi wacomadra: il faut aller prier au culte le dimanche sous peine d'être puni.

Fitiku kale kale tro së kejë eё tro sa hane si ke tru catre la dro së qa caha ngöne helep.

« Fitiku kale kale, allons vers le littoral, allons nous baigner à la mer, nous sommes trop sales en revenant du champ. »

Neköi wacomadra a sixane la sabath ngo e thupenehmi fenesi hë mele i hmunë.

" La petite fauvette sous-estime le culte du dimanche, mais le lundi elle est morte. »

Malgré l'expression et l'intention des informatrices de s'attacher fidèlement aux traditions, ce 
répertoire reflète une grande part de modernisme. La tradition orale est le moyen d'inscrire une pratique chantée à la fois dans la continuation et dans la modernité. Ces expressions orales chantées reflètent ainsi un parfait équilibre entre tradition, reformulations d'expression du passé dans le présent.

\section{Un répertoire très évolutif : une double reformula- tion permanente}

Bien que ce répertoire pour enfants soit qualifié de traditionnel, les différentes caractéristiques énumérées ci-dessus permettent de le définir comme un système très ouvert, très changeant et évolutif. Il existe alors, dans le répertoire chanté que les adultes adressent oralement aux enfants, une multitude de références collectives, individuelles, anciennes et contemporaines, divergentes et communes, variantes et permanentes, traditionnelles et modernes qui sont autant de micro-variations et de combinaisons possibles.

L'interprétation des chants et jeux chantés que les femmes adressent aux enfants se réalise dans une structure à la fois constante et variable. Ce répertoire ne s'inscrit donc pas dans un circuit fermé, dans une logique limitée, figée. Il s'affirme bien plutôt comme un système très évolutif, très souple et très ouvert, capable d'intégrer des éléments exogènes nouveaux ou événementiels et d'un renouvellement perpétuel sans pour autant changer de fonctionnement.

Cette caractéristique trouve une justification directement dans le groupe producteur de ces chants et jeux chantés, dans le fait que les vieilles femmes font pénétrer et retranscrivent la culture englobante, les préoccupations et les événements d'une époque, d'un moment, en une zone géographique plus ou moins élargie, d'une communauté et d'une famille, dans les chants qu'elles adressent à une autre génération. C'est pourquoi chaque femme exprime dans ces chants et jeux chantés pour enfants, dans un contexte sociohistorique précis, produit en un endroit historiogéographique particulier, ce qui fait partie de son environnement. Elle y greffe une partie d'ellemême et est influencée par ses propres expériences, par des événements marquants de sa vie, par des préoccupations plus ou moins personnelles. En ce sens, le chant est riche de microdivergences culturelles. Les chants et jeux chantés pour enfants collectés sur ce terrain de Lifou sont donc les expressions, les représentations, les symbolisations et les conceptions d'une réalité plus ou moins partagée par l'ensemble des mem- bres de la communauté, correspondant à un moment donné particulier par un individu bien précis, en un point géographique ciblé. On comprend alors l'importance des données temporelles, géographiques et identitaires dans ce répertoire.

Chaque femme remodèle son propre passé, le relit, le reformule aujourd'hui pour l'inscrire dans le présent. Ce répertoire constitue une première éducation, la transmission d'un bagage culturel significatif dans la socialisation et la construction de l'identité de l'enfant. Il véhicule ainsi une certaine actualité, s'adaptant, se remodelant au gré des besoins d'expression. Il exprime la combinaison d'une certaine lecture du passé avec l'assimilation d'éléments nouveaux très contemporains. Les chants pour les enfants sont donc l'expression d'une double formulation permanente : les grands-mères transmettent une certaine reformulation de préoccupations individuelles et/ou collectives, une re-lecture de faits anciens et contemporains, une explication et une interprétation d'une vision du monde aux configurations hétéroclites, tout en s'inscrivant dans une pratique musico-culturelle dans laquelle tous peuvent se reconnaître, en réactualisant un passé indéfini dans un présent particulier, de la mémoire et de la tradition transmise par leurs ancêtres.

Les chants et jeux chantés pour enfants deviennent alors un carrefour de perceptions, de tradition, de modernité, voire même de confrontations culturelles. Ils s'érigent donc comme la traduction de leur expansion et de leur interpénétration. Car comme le soulignent Alban Bensa et Isabelle Leblic dans l'introduction de l'ouvrage collectif En pays kanak:

«Les travaux regroupés dans ce volume montrent, chacun à leur manière, que le changement éclaire les permanences. » (Bensa et Leblic, $2000: 5$ )

Ils reflètent ainsi les rapports de l'interprète avec sa société, sa culture et l'histoire de son temps, aux configurations multiples et variables.

\section{RÉFÉRENCES BIBLIOGRAPHIQUES}

Ammann Raymond, 1997. Danses et musiques kanak, Nouméa, ADCK.

BENSA Alban, 1998, Nouvelle-Calédonie : Vers l'émancipation, Paris, Découvertes Gallimard.

Bensa Alban et Isabelle LeBLIC (dir.), 2000. En pays kanak, Paris, éditions de la maison des sciences de l'homme.

BRIL Isabelle, 2000. Enquête linguistique et enjeux culturels, in Alban Bensa et Isabelle Leblic (dir.), En 
pays kanak, Paris, Éditions de la maison des sciences de l'homme, pp. 273-291.

FrIEDMAN Jonathan, 2002. Y a-t-il un véritable hawaiien dans la salle ?, in Christine Hamelin et Éric Wittersheim (dir.), La tradition et l'État. Églises, pouvoirs et politiques culturelles dans la Pacifque, Paris, L'Harmattan, Cahiers du Pacifique Sud contemporain 2, pp. 207-245.

LEPOUTRE Marie, 2000. Le pluralisme médical à Lifou, in Alban Bensa et Isabelle Leblic (dir.), En pays kanak, Paris, Éditions de la maison des sciences de l'homme, pp. 293-309.
PAINI Anna, 2003. Rhabiller les symboles : les femmes kanak et la robe mission à Lifou (NouvelleCalédonie), in Isabelle Leblic (dir.), NouvelleCalédonie 150 ans après la prise de possession, Journal de la Société des Océanistes 117, Paris, pp. 233253.

TuibaOU Jean-Marie, 1984. La présence kanak, in Alban Bensa, Nouvelle-Calédonie : Vers l'émancipation, Paris, Découvertes Gallimard, pp. 159-161. 


\title{
MISCELLANÉES
}

\section{La semaine d'anthropologie audiovisuelle sur l'Océanie'. Parcours d'une doctorante en ethnomusicologie au cœur de cet événement}

par

\author{
Stéphanie GENEIX-RABAULT*
}

\section{RÉSUMÉ}

Cet article retrace le déroulement d'une semaine de colloque sur l'anthropologie audiovisuelle en Océanie, au travers de ma propre perception en tant que doctorante en ethnomusicologie-musicologie. Il tente de mettre en exergue l'intérêt du film ethnographique dans la démarche classique de collecte d'informations, dans la restitution du vécu du chercheur sur le terrain et dans la présentation des résultats scientifiques obtenus. La diversité des films projetés reflète les multiples configurations possibles des documentaires, tant dans l'étape de prises de vue que dans la phase de montage. J'examine alors de manière plus détaillée certains films marquants de cette semaine, pour tenter de me situer par rapport à mes propres images et recherches.

MotS-CLÉS : anthropologie visuelle, film ethnograhique, collecte de données, description ethnographique, ethnomusicologie, Océanie, Lifou.

\begin{abstract}
This article relates the march of a one-week symposium about audiovisual anthropology in Oceania, through my owm realization as an ethnomusicologymusicology doctor's degree's student. It tends to show up the ethnographic movies's interest in the datum collecting classical process, in the restitution of the research worker's personal experience in the land and in the presentation of scientific results obtained. The projected movies diversity reflects the documentaries multiple possible forms, in shooting as in editing. Then, in a deeper way, I examine some out-standing movies of this week, to try to place myself about my pictures and investigations.
\end{abstract}

KEYwORDS: audiovisual anthropology, ethnographic film, datum collecting, ethnographic description, ethnomusicology, Oceania, Lifou.

* Doctorante en ethnomusicologie-musicologie, Paris IV-LACITO-CNRS, sgeneix@hotmail.fr 
Du 5 au 9 décembre 2005 s'est déroulée la semaine d'anthropologie audiovisuelle sur Le film en Océanie au CNRS-images à Meudon. Ce colloque a été mis en place grâce à la volonté de la Société des Océanistes de relancer ses cycles de conférences selon une nouvelle version, plus attractive pour le public. C'est ainsi que Lorenzo Brutti a contacté Nathalie Lambert, du CNRS-images, et le Comité du film ethnographique pour mettre sur pied cette opération. Et il faut remercier la ténacité de Lorenzo et de Nathalie pour la réalisation de cette semaine. Dans la salle du pavillon de la communication, une vive émotion et un intérêt particulier se sont emparés de l'auditoire avec la présence d'éminents spécialistes des cultures océaniennes. En tant que doctorante en ethnomusicologie et néophyte en la matière, plusieurs attentes motivaient ma présence à ce colloque.

L'intérêt vivant et sans cesse renouvelé, aux configurations hétéroclites, des films réalisés dans divers endroits de cette zone géographique océanienne font partager la pluralité de ces cultures et des approches visuelles envisageables. Un grand nombre de films ethnographiques de genres très différents a été présenté, en soulignant qu'il n'existe pas qu'une seule règle de conduite pour réaliser un documentaire ethnographique. L'anthropologie visuelle ne fonctionne pas comme une œuvre close obéissant à des lois formelles strictes et figées, mais bien plutôt comme l'empreinte visible de partis pris du chercheur et/ou du réalisateur, du type de relations qu'il a pu établir avec les protagonistes culturels et des intentions de l'auteur. Il transparaît dans l'image une partie de la propre sensibilité et de la personnalité du réalisateur/chercheur. Entre le filmeur et le filmé s'établit une interaction singulière qui peut prendre plusieurs configurations : de l'échange à l'inversion des rôles, jusqu'à l'instrumentalisation de l'investigateur.

Loin des documentaires télévisés esthétiquement plaisants, agréables et fascinants, aux images aseptisées, les films proposés reflétaient bien au contraire un engagement réel du chercheur, mais aussi des protagonistes culturels de la société observée. Les différents intervenants de cette semaine ont par ailleurs souligné trois applications de l'outil audiovisuel, envisagé dans un premier temps comme un instrument de recherche et d'analyse sur l'homme et les sociétés de tradition orale étudiés, puis dans un second temps comme un document complet pouvant servir la description ethnographique, enfin comme un support permettant de compléter la collecte de données.

\section{L'intérêt du film ethnographique dans la démar- che scientifique}

À la fois conçu comme la présentation et la retransmission des résultats d'une recherche effectuée, de ce qui est observé sur le terrain par le chercheur et/ou le réalisateur, mais aussi comme instrument de recherche, c'est-à-dire comme un outil permettant de renouveler autant de fois que nécessaire son observation directe, et d'aller encore plus loin dans la démarche de collecte d'informations, le film ethnographique sort grandi dans sa diversité d'expression de configurations possibles et dans l'intérêt qu'il apporte à toute démarche scientifique. La prise d'images offre la possibilité de revenir en permanence sur l'ensemble des faits étudiés, de réitérer ou de renouveler à volonté une bonne partie de l'observation. Dans la démarche classique d'enquête, le chercheur travaille à partir de son carnet de notes, sans toujours avoir la possibilité de retourner sur le terrain pour vérifier et/ou approfondir certaines données.

De plus, le film permet de témoigner de la vivacité d'une culture dans sa globalité. Il regroupe une multitude de détails complexes : les discours, l'ensemble des paroles, les chants, les gestes, les attitudes, les sons, les expressions, les postures, les déplacements... Autant d'éléments environnant les pratiques culturelles qui sont significatifs. L'image permet de capter une multitude de détails qui sont en réalité très importants et extrêmement difficiles à décrire. Le document ethnographique de type classique est cohérent. Néanmoins, le film permet d'aller plus loin dans la compréhension, dans l'analyse et la restitution. Il se présente comme un matériau complet, renvoyant toute une série de données du vécu ethnographique, que l'écrit a quelquefois du mal à exprimer. Grâce à ce document doté d'une épaisseur particulière, le chercheur peut détailler très précisément un savoir, une pratique, des attitudes.

Aussi permet-il d'aller plus loin dans l'analyse en offrant la possibilité d'obtenir des informations verbales et non verbales, formelles et informelles sur le sujet étudié en tant que support visuel $\mathrm{du}$ comportement non verbal. Cette méthode de collecte et d'analyse des données est intéressante à intégrer dans le processus d'observation et de recueil des informations sur le terrain. Elle permet d'élargir et de diversifier la palette de perception, dans les émotions, les gestes, les postures, les interactions entre différents paramètres, quand l'écrit a quelquefois du mal à le faire. 
Outre l'intérêt riche de ce support dans les perspectives d'observations directes, il sert également la description ethnographique. Il se présente comme une méthode de restitution précise, globale et complète, puisqu'elle regroupe l'interconnexion de la quasi totalité de l'expérience vécue par le chercheur sur le terrain et préserve plus ou moins la simultanéité de l'événement. Elle permet d'enregistrer des détails complexes de l'interaction non verbale d'une pratique et de sa réalisation, au travers de l'image, et d'en extraire des connexions significatives.

De plus, et cela a été maintes fois souligné tout au long de la semaine, le film est un support illustratif fabuleux, l'image portant en ellemême des choses qu'il est impossible de décrire, de restituer par écrit. Elle permet de découvrir, de refléter une société de manière analogue aux descriptions d'expériences de terrain, mais d'une façon plus complète. Elle présente une vision de la vie sur le terrain telle que les chercheurs l'ont vécue, sans ornements, en recherchant l'histoire à partir du quotidien pour mettre l'essentiel en valeur. Le chercheur/réalisateur, dans cette démarche audiovisuelle anthropologique, doit trouver son propre langage cinématographique pour rendre au mieux la réalité observée, au même titre qu'un auteur structurant son texte, utilisant des figures de style et des expressions de langage pour servir sa description ethnographique écrite.

L'image permet non seulement d'aller plus loin dans la démarche descriptive tant elle se structure à partir de l'audio-visuel lui-même, mais aussi dans la collecte de nouvelles données une fois l'observation effectuée. Visionner le film avec des spécialistes ou des protagonistes de cette culture permet d'obtenir plus de détails et de discours sur ce que l'individu lui-même ou les autres sont en train de faire. Non seulement le film peut être relu autant de fois que nécessaire, mais il est possible de le faire relire avec d'autres personnes ou avec ceux sur lesquels le documentaire portait. S'appuyer sur ce type de projection permet d'approfondir le sujet en posant de nouvelles questions et d'obtenir d'autres informations extrêmement précises.

En ce sens, ce support complète la démarche classique de collecte par observation directe et participante, par entretiens classiques et prises de photographies. Il n'est pas non plus question d'opposer le document audio-visuel à la description de type ethnographique ou à la parole, mais il s'agit plutôt de considérer ce moyen d'expression et de collecte comme complémentaire. Bien que l'entretien fournisse l'essentiel du matériel d'analyse, le film n'est pas dénué d'intérêt. Mais il ne se substitue pas non plus à la méthode d'entretien classique de collecte d'information. Il vient seulement compléter la démarche d'enquête de terrain de type ethnographique.

Ces généralités d'intérêt dans toute recherche scientifique ne doivent pas pour autant faire oublier les diverses possibilités d'exploration de cet outil et les multiples questions qu'il suscite, tant sur la relation du chercheur et des autochtones avec la caméra que sur celles liées aux prises de vue et au montage :

«Comment s'engager réellement sur le terrain avec une caméra ?»

« Comment retranscrire visuellement des réalités d'expériences de terrain faites dans différentes situations : au cours d'observations directes, actives et participantes, ou d'entretiens libres ou semi-directifs?»

"Que doit observer et filmer le chercheur?»

«Comment observer et filmer le réel du terrain?»

« Par la suite, comment le présenter, comment le rendre réel sans le dénaturer au moment du montage? »

«Comment refléter sa rencontre et son expérience avec l'autre?»

«Faut-il une équipe pour réaliser le documentaire parfait?»

«Faut-il filmer en intégralité ou privilégier les micro-séquences?»

« Faut-il conserver en permanence un œil derrière l'objectif ou un regard hors du champ pour saisir ce qui se passe en amont ?»

«En quel cas faut-il balayer le champ ?»

«Faut-il filmer en plans larges ou serrés?»

\section{Le déroulement de la semaine}

Le choix des films, remarquable par sa diversité et sa densité, agencé par thématique tout au long de la semaine, a suscité un débat riche et varié sur les préoccupations fondamentales liées à cette discipline. Après chaque projection, des discussions animées entre le/les intervenant(s) et un auditoire attentif et intéressé ont été menées et parfaitement orchestrées par l'ethnologue Lorenzo Brutti. Elles permettaient d'accéder à un autre niveau de compréhension des intentions, des préoccupations, des difficultés et des limites de l'auteur au sujet du film présenté. Ce fut l'occasion idéale d'obtenir de nouvelles données sur la manière d'aborder cet outil et sur son intérêt dans la démarche d'enquête de terrain de type ethnographique.

La sélection proposée permettait de mettre l'accent tout au long de cette semaine sur des 
préoccupations de fond et de forme. Différents regards comparés sur des zones d'investigations proches et/ou lointaines, dirigés sur les mêmes objets et/ou sur des sujets contigus ou divergents, sur les similitudes et les diversités culturelles, sur les constantes et les évolutions en Océanie, ont permis la confrontation d'opinions différentes. Et plutôt que de tenter de présenter des généralités de systèmes, tous ces films se sont attachés à la description d'individualités, de partis pris plus ou moins personnels, tout en mettant en exergue une constante dans cette démarche : l'impérativité du travail prolongé sur le terrain et l'invariante préoccupation de présentation d'une réalité vécue par le chercheur/réalisateur et/ou les témoins eux-mêmes.

\section{Les différentes configurations des films proposés}

Les projections d'extraits de séquences filmées, Planète Baruya de Maurice Godelier et Loin des médias : la vidéo comme carnet de notes complémentaires. Recherches en cours sur les rites masculins ankave (Papouasie Nouvelle-Guinée) de Pierre Lemonnier, ont retracé des rites d'initiation masculins filmés auprès de deux communautés différentes de Papous en NouvelleGuinée. Dans ce cas précis, le support audiovisuel se présente comme un " carnet de notes complémentaire » dans les démarches d'enquête et de description de types ethnographiques. Il offre en effet la possibilité à ces deux anthropologues, une fois les investigations menées, de pouvoir comparer deux objets de recherche similaires, en deux points géographiques différents d'un même territoire, auprès de deux communautés appartenant au même groupe géographico-ethnique.

Pour Pascale Bonnemère, Démocratie héliportée en Papouasie Nouvelle-Guinée : l'apprentissage d'un rituel électoral (juin 1997), le documentaire retranscrit une réalité authentique d'expérience de terrain. Elle a présenté quinze minutes denses de prises de vue permettant au spectateur de s'imprégner de l'atmosphère réelle du déroulement d'une initiation électorale « héliportée " dans des lieux reculés de Papouasie Nouvelle-Guinée. Le film, sans commentaires en voix-off, sans effets, sans fioritures, rend compte le plus simplement possible de la réalité des circonstances de déroulement des élections des députés de Provinces et du Parlement sur ce territoire. Autour de cette même thématique, l'ethnologue Éric Wittersheim a présenté un autre type de film, Grassroots, ceux qui votent, réalisé par entretiens libres ou semi-directifs. En confrontant les points de vue de différents individus sur ce sujet, il retrace le déroulement de la campagne et des élections législatives au Vanuatu. Au travers d'un échantillon large, riche et varié de propos d'informateurs, cet auteur aborde une autre configuration qui tente elle aussi d'être la plus représentative possible d'une réalité de déroulement électoral.

Le film plein d'humanité Le ciel dans un Jardin du chercheur Stéphane Breton propose un autre genre de documentaire. Il retrace, sous la forme d'un récit scientifique cinématographique riche, émotionnel et esthétique, le quotidien de l'investigateur qui dialogue et partage celui d'une communauté de Papous. Les commentaires en voixoff se concentraient essentiellement sur l'émotion vécue par le chercheur lui-même. Une nouvelle manière de retransmettre l'expérience est présentée tout en mettant en avant un point essentiel dans la démarche anthropologique visuelle. Il reflète l'importance de s'immerger complètement dans la société observée, de développer des relations de confiance avec ses protagonistes et insiste sur le temps, la patience qu'exige un tel processus, ainsi que sur l'investissement affectif du chercheur. Outre ces contraintes, Stéphane Breton a largement souligné l'intérêt de ce support dans la démarche scientifique de collecte d'informations. Il lui a permis de faire des conquêtes ethnographiques et des avancées ethnologiques considérables, notamment en linguistique. En le visionnant avec les individus sur lesquels le film portait et avec d'autres spécialistes, les commentaires émis ont permis à ce chercheur de collecter d'autre vocabulaire, d'élucider le sens de certains mots encore obscurs, d'accéder à un autre niveau de compréhension... L'image se suffisant à elle-même, elle permet de rendre compte de l'expérience de terrain comme si le spectateur la vivait, quand l'écrit a quelquefois des limites.

Contrairement à l'expérience de Stéphane Breton, à la fois chercheur et réalisateur, passant dix heures par jour sur le terrain avec sa caméra, d'autres types de documentaires trouvent un accomplissement dans des collaborations entre chercheurs et cinéastes. Les réalisations s'effectuent dans diverses conditions et résultent de choix personnels. Dans Les esprits du Koniambo, Alban Bensa et Jean-Louis Comolli procèdent par entretiens préparés, quelquefois répétés plusieurs fois, plaçant le chercheur au cœur même de l'image. L'ethnologue s'investit sans avoir peur de se montrer. Son film tente de restituer les relations qu'il a pu tisser avec certains informateurs depuis trente ans de recherche sur ce terrain, tout en retranscrivant une partie d'enquête 
sur leurs préoccupations contemporaines sur le projet de mine dans le Nord. Dans Lettres aux morts, André Itéanu et Etan Capon quant à eux retracent, sans aucune préparation d'entretiens, la perception des chercheurs occidentaux en Papouasie Nouvelle-Guinée et leur instrumentalisation par les autochtones.

Ces deux types de documentaires mettent en exergue les contraintes de réalisation de films dans le cadre de collaborations entre cinéastes et chercheurs. Ils attestent de la possibilité d'une telle expérience pluridisciplinaire entre une équipe de cinéma et un chercheur scientifique. Ils mettent l'accent sur la complémentarité des deux points de vue dans cette démarche, tout en soulignant la divergence d'approche du terrain, les difficultés, les limites et les fusions de chacun dans ce projet commun. Cette aventure n'est envisageable que lorsqu'une relation de confiance entre le chercheur, le réalisateur et les protagonistes culturels de la société observée est installée. Elle engendre une certaine prise de risques partagée. Le cinéaste, ignorant des contraintes et des exigences culturelles de la population observée, doit complètement se reposer sur l'ethnologue. Inversement, le scientifique n'est pas maitre des prises de vue qui sont faites sur le terrain.

Un autre genre est proposé par Séverin Blanchet et Martin Maden dans Kantri Bilong Yumi, la Papouasie de la famille Maden: le cinéma direct, orienté par et sur des questions de famille. Ce film place la parole des protagonistes culturels au cœur même du documentaire et de sa réalisation, en retraçant le récit et les échanges personnels d'un Papou avec sa famille. Le chercheur n'a ici qu'un rôle secondaire puisque les prises de vue tournent autour du regard d'une famille, tout en abordant une destinée individuelle, collective, ancienne et contemporaine.

Le documentaire d'observation ethnologique de Lorenzo Brutti reflète un savoir-faire incontestable de la technique de prise de vue, combiné avec une grande maîtrise esthétique de cet outil. Son film, Keva et Delphine, offre un confort esthétique tout à fait subsidiaire mais qui permet au spectateur de s'installer dans une ambiance profondément chaleureuse et agréable, tout en donnant à réfléchir sur les questions de confrontation culturelle et de reconstruction patrimoniale, dans le cadre du mariage d'une anthropologue belge avec un Pascuan.

Dans le même genre, les films d'observations directes menées par Marc Tabani, Alors vint John Frum : une tragédie culturelle des Mers du Sud, et celui de Thomas Balmès et Lorenzo Brutti, L'Évangile selon les Papous, retracent le syncré- tisme religieux existant en Océanie sur deux territoires différents : le Vanuatu et la Papouasie Nouvelle-Guinée. Le documentaire s'inscrit dans ce cas-là comme le témoin privilégié d'un tournant socio-historique de toute importance de sociétés en pleine mutation.

Pour Barbara Glowczewski et Wayne Jowandi Barker, Quête en terre aborigène propose un voyage audiovisuel interactif avant-gardiste. Outre l'aspect novateur de l'utilisation de ces nouvelles technologies, la démarche de réalisation des prises de vue pour ces deux chercheurs, comme pour Jessica de Largy Healy dans Galiwin'ku indegenous knowledge center (Terre d'Arnhem), s'inscrit dans une autre perspective. Réalisée sur demande des Aborigènes, elle reflète le désir des autochtones d'exprimer et de conserver des choses importantes. Les images prennent dans ces circonstances une valeur d'archive familiale. Collectées sur demande des protagonistes culturels, elles servent à partager et à conserver un certain savoir, une certaine pratique, pour les générations futures. Il ne s'agit pas là de fixer des éléments en voie de disparition, car la mémoire est toujours bien vivante, mais plutôt de témoigner d'une pratique en un temps donné.

Une autre application de l'anthropologie visuelle a été abordée par Marika Moisseeff, qui a proposé une intervention innovante, Nature contre Culture ou le pouvoir animalisant de la viviparité, s'inscrivant dans une démarche de décodage et d'analyse de séquences de films de science-fiction. Dégageant différents niveaux d'interprétation d'images sélectionnées, elle a ainsi démontré que l'anthropologie visuelle ne concerne pas exclusivement la démarche scientifique d'enquête de terrain ethnographique, mais qu'elle peut tout aussi bien s'appliquer à des genres qui font partie de notre quotidien cinématographique.

Les disciplines concernées par le film ethnographique s'élargissent. Les ethno-archéologues Pierre et Anne-Marie Petrequin, les chercheurs Michel et Catherine Orliac, ainsi que Rosa Olmos, et moi-même ethnomusicologue, restituent avec ce support une partie de leurs résultats scientifiques ; le linguiste Alessandro Duranti et Jean-Michel Chazine s'appuient sur ce matériau comme un outil d'analyse. Tous démontrent l'intérêt du document visuel ethnographique dans la démarche scientifique en tant que support restituant une partie de l'expérience, des résultats de recherche et comme outil d'analyse. C'est donc sous le signe de l'interdisciplinarité que se plaçait cette semaine du film en Océanie. 


\section{Les motivations de ma participation}

Tous ces films reflètent l'investissement du scientifique sur le terrain, mettent en exergue l'importance et la nécessité des relations de complicité, de proximité, de confiance et d'intimité avec ceux qui sont filmés. Le résultat final qui en découle provient d'une grande variété de facteurs subjectifs, objectifs, de parti pris plus ou moins individuels et/ou collectifs. Cela dépend aussi en partie des orientations scientifiques et esthétiques choisies par le réalisateur et/ou le chercheur et/ou le protagoniste culturel luimême. D'autres facteurs entrent encore en ligne de compte, les questions financières liées aux budgets attribués pour la réalisation des films, les moyens techniques... Tous ont permis de réfléchir à différents aspects de la réalisation de films ethnographiques dans la démarche scientifique de recherche, ainsi que sur les questions d'ordre moral et légal posées par leur réalisation et leur exploitation. Les œuvres présentées ont mis en relief la multiplicité des choix individuels possibles et des orientations envisageables. Chacune a reflété l'investissement du chercheur à se détacher des documentaires télévisés trop lissés, largement stéréotypés, et a permis de prouver qu'il existe de multiples façons de réaliser et de monter un film.

Plusieurs préoccupations personnelles, directement liées à mes précédentes expériences de terrain, m'ont incitée à participer à cet événement. J'ai réalisé, depuis cinq ans, quatre missions sur Lifou, une des quatre îles Loyauté de Nouvelle-Calédonie ${ }^{2}$, représentant au total dix-huit mois d'investigation sur ce terrain. Doctorante en ethnomusicologiemusicologie à Paris IV-LACITO-CNRS, je prépare une étude sur Les chants et jeux chantés pour enfants en Lifou ${ }^{3}$. Au-delà de l'intérêt que je porte à cette aire géographique océanienne, je me suis moi-même essayée lors de ma dernière investigation $^{4}$ à la réalisation de prises de vues sans aucune formation technique préalable. Néophyte en la matière et livrée à moi-même, certaines interrogations se sont présentées au cours de mon enquête sur le terrain, quant à la meilleure méthode à adopter pour effectuer les prises de vue.

Après de multiples tentatives qui me semblaient infructueuses, je me suis rapidement fixé quelques directives de conduite : j'ai choisi de filmer en plans larges et fixes pour capter un maximum d'éléments sans fractionner les prises de vues, en enregistrant l'intégralité de l'action observée. De retour de mission, je me suis attelée à la réalisation d'un documentaire sur Les chants et jeux chantés de Lifou ${ }^{5}$. Le dérushage ${ }^{6}$ des quinze heures de prises de vue effectué au préalable, puis le montage final d'un film de 26 minutes, suscitent alors un certain nombre de nouvelles interrogations sur l'utilisation de cet outil et la scénarisation du film ethnographique.

J'attendais de cette semaine d'anthropologie audiovisuelle en Océanie qu'elle me permette d'avoir accès aux différentes configurations possibles de réalisation de film. J'aspirais à rencontrer des spécialistes dans ce domaine qui pourraient me donner des conseils techniques et méthodologiques pour améliorer mes prises de vue, le documentaire que j'ai pu réaliser, et toutes les préoccupations qui en découlent. J'y cherchais des réponses aux interrogations que je me pose à différents niveaux sur ce sujet : tant sur des questions liées à la relation du chercheur et des autochtones avec cet outil que sur celles liées aux prises de vue et au montage...

Ce sont vraisemblablement ces préoccupations fondamentales qui m'ont poussée à participer activement à cet événement. J'y attendais avant tout des réponses, des solutions concrètes et pertinentes liées à mes interrogations.

La vision et la projection du documentaire réalisé ont suscité plusieurs réflexions. D'un point de vue technique, certains ont souligné le manque de formation préalable, tant en milieu universitaire que dans la recherche, à l'utilisation des nouveaux outils de prises de son et de vue pour les jeunes chercheurs. J'avais moi-même abordé cette regrettable lacune en relatant les difficultés techniques auxquelles j'avais été confrontée une fois sur le terrain avant la projection. Certaines solutions ont alors été apportées

2. Ce territoire se situe dans la partie sud-ouest de l'Océan Pacifique. Il appartient au groupe géographique de la Mélanésie.

3. «Nyima me elo thatraqai haa nekönatr ngöne la qene drehu : chants et jeux chantés pour enfants en Lifou (Îles Loyauté,Nouvelle-Calédonie). L'expression d'un répertoire en renouvellement permanent », doctorat d'ethnomusicologie-musicologie, préparé à Paris IV-Sorbonne-LACITO-CNRS, sous la direction d'André-Marie Despringre.

4. Une dernière enquête de terrain de vérifications d'hypothèses a été menée très récemment, du 31 décembre 2004 au 8 mars 2005.

5. Nyima me elo thatraqai haa nekönatr ngöne la qene drehu: chants et jeux chantés pour enfants en Lifou, novembre 2005 , 26 minutes, présenté le 9 décembre dans le cadre de ce colloque sur le Film en Océanie.

6. Les quinze heures de rushes réalisés englobent : une heure de film lors de l'intronisation d'un pasteur ; une heure de représentations de danses ; une heure de préparatifs de mariage ; trois heures de vie quotidienne ; trois heures de baptême ; deux heures de film sur un petit îlot; quatre heures sur ce répertoire et sur les pratiques de maternage. 
pour tenter d'améliorer non seulement les prises de vue, mais aussi le montage audio-visuel :

- Préférer aux planches de texte un commentaire en voix-off; sinon, en normaliser la durée à 5 secondes ;

- Envisager d'effectuer les prises de vue avec deux caméras pour pouvoir filmer en plans plus serrés la gestuelle des enfantines pendant que l'autre caméra enregistre en plans larges et fixes.

Du point de vue du contenu du documentaire, le public a largement pointé du doigt l'intérêt primordial de ce répertoire oral chanté pour enfants en tant que processus éducatif et socialisant. Il a engendré quelques pistes de réflexion :

- Comment en expliciter un peu plus le contenu?

- Quel rôle et quelle place prend-il au sein de la société traditionnelle en tant que système éducatif?

- Quelle part prend ce répertoire dans le système scolaire du territoire?

Aussi cet événement a-t-il largement stimulé une réflexion approfondie sur cette pratique, sur cet outil, sur la diversité de méthode de réalisation de l'autre, ainsi que sur ses multiples facettes possibles. En ce sens, il a proposé de poser les marques liées à une pratique d'anthropologie audiovisuelle, aux dispositifs à mettre en œuvre pour approcher, comprendre, donner à voir et à entendre, dans leur intime complexité et dans leur diversité, des hommes et des femmes aux prises avec les réalités de la recherche sur ce terrain océanien. Le film ethnographique a pris pour moi une autre dimension : il s'est présenté comme un instrument naturel de collecte et de découverte d'information, d'exposition des résultats de l'enquête de terrain de type ethnographique, au même titre que les autres outils avec lesquels j'ai l'habitude de travailler. Le film est un moyen d'expression complet et le support d'une réflexion riche sur le réel du vécu de l'expérience ethnographique de terrain du chercheur. Cette semaine d'anthropologie visuelle en Océanie a fait germer dans mon esprit de nouveaux projets d'investigation sur le Lifou.

Enfin, la participation à ce colloque a largement stimulé ma réflexion quant à mes propres recherches sur le terrain. Elle m'a offert la possibilité de confronter les images et les résultats des différents intervenants aux investigations personnelles menées sur cette zone géographique. Ce colloque donnait l'opportunité inouie d'avoir un aperçu plus global, plus « océanien », de pra- tiques culturelles divergentes et similaires, en se projetant sur des terrains sur lesquels je n'ai pas eu l'occasion d'aller et dont je n'ai qu'une connaissance fragmentaire.

\section{En guise de conclusion}

Merci pour ce voyage audiovisuel océanique incroyable! Il existe une réelle demande et attente de la part des étudiants qui souhaitent se lancer dans ce genre d'expérimentation, d'exploration. En atteste leur présence tout au long de la semaine. Tous ont souligné que ce genre de formations aux nouvelles technologies audiovisuelles se fait trop rare dans le cursus universitaire et scientifique. Bien trop souvent, le jeune chercheur est livré à lui-même sur le terrain. Aussi ce genre de manifestations est-il primordial pour tous ceux qui s'intéressent de près ou de loin à ce genre de réalisation.

\section{BIBLIOGRAPHIE}

Ateliers d'ethnomusicologie, Cahiers de musiques traditionnelles, Genève, Georg éditeur.

Belmont Nicole et Jean-François Gossiaux, 1997. De la voix au texte, l'ethnologie contemporaine entre l'oral et l'écrit, Paris, Éditions du cTHS.

BLACKING John, 1967. Venda children's songs, Witwatersrand University Press, Johannesburg.

—, 1977. «L'homme producteur de musique », Ethnomusicologie 10, Paris, Seuil, pp. 108-116.

DeSPRINGRe André-Marie, 1993 (9-10 décembre). «Propriété intellectuelle, droits d'auteur et droits voisins dans la recherche sur la mémoire orale des communautés : réaction d'un anthropologue à la recommandation de l'UNESCO de 1989 », La Recherche éthique et les sciences, Colloque interdisciplinaire du Comité national de la Recherche scientifique, pp. 459-469.

GoODy Jack, 1994. Entre l'oralité et l'écriture, Paris, PUF, Ethnologies.

Lallemant Suzanne (dir.), 1987. L'ethnologue et son terrain, Paris, Association française des anthropologues, pp. 29-30.

LAMBert Jean, 1996. «Ceux qui n'étaient pas là ne pourront jamais comprendre... une ethnomusicologie sans magnétophone? ", Cahiers de musiques traditionnelles 8, Genève, Ateliers d'ethnomusicologie, pp. 85-103.

LAPLANTINE Francois, 1996. La description ethnographique, Paris, Nathan université, Sciences sociales.

LENCLUD Gérard, 1986, «En être ou ne pas en être : l'anthropologie sociale et les sociétés complexes », L'Homme 97-98, pp. 143-153. 
LÉvi-Strauss Claude, 1983. L'identité, Paris, PUF, Quadrige.

MASQuelier Bertrand et Jean-Louis Siran, 2000. Pour une anthropologie de l'interlocution, Réhtoriques du quotidien, Paris, L'Harmattan, Logiques sociales.

Mauss Marcel, 1992. Manuel d'ethnographie, Paris, Payot.

MWÀ VÉÉ 39, 2003. Quand la parole s'exporte, Nouméa, ADCK.
MWÀ VÉÉ 43, 2004. De la parole à l'image, Nouméa, ADCK.

RougET Gilbert, 1968. « L'enquête d'ethnomusicologie », Ethnologie générale, Paris, La pléiade, pp. 333-348.

TERRAIN, Carnets du patrimoine ethnologique, Paris, Mission du Patrimoine ethnologique.

Verdier Yvonne, 1995. Coutume et destin. Thomas Hardy et autres essais, Paris, Gallimard NRF. 